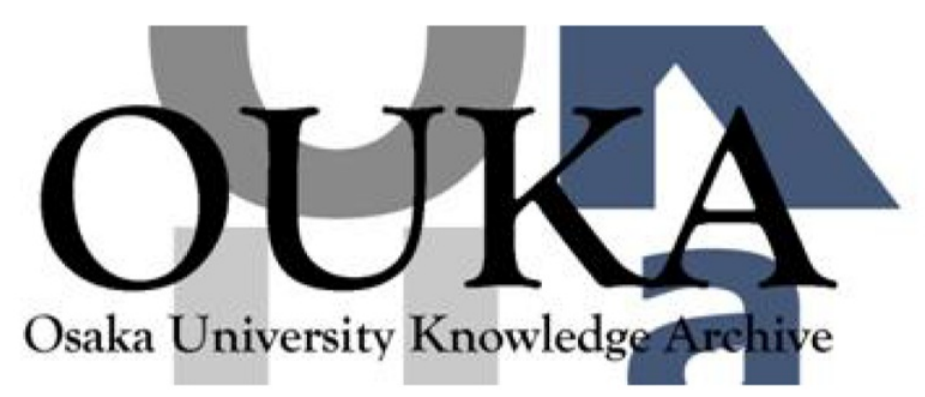

\begin{tabular}{|c|l|}
\hline Title & $\begin{array}{l}\text { Electro-tunable laser action in a dye-doped } \\
\text { nematic liquid crystal waveguide under } \\
\text { holographic excitation }\end{array}$ \\
\hline Author(s) & $\begin{array}{l}\text { Matsui, Tatsunosuke; Ozaki, Masanori; Yoshino, } \\
\text { Katsumi }\end{array}$ \\
\hline Citation & Applied Physics Letters. 83(3) p. 422-p. 424 \\
\hline Issue Date & 2003-06-16 \\
\hline oaire:version & VoR \\
\hline URL & https://hdl.handle.net/11094/75829 \\
\hline rights & \\
\hline Note & \\
\hline
\end{tabular}

Osaka University Knowledge Archive : OUKA

https://ir. Library. osaka-u. ac. jp/

Osaka University 


\section{Electro-tunable laser action in a dye-doped nematic liquid crystal waveguide under holographic excitation}

Cite as: Appl. Phys. Lett. 83, 422 (2003); https://doi.org/10.1063/1.1593827

Submitted: 10 March 2003. Accepted: 30 May 2003. Published Online: 16 July 2003

Tatsunosuke Matsui, Masanori Ozaki, and Katsumi Yoshino

ARTICLES YOU MAY BE INTERESTED IN

Electrically color-tunable defect mode lasing in one-dimensional photonic-band-gap system containing liquid crystal

Applied Physics Letters 82, 3593 (2003); https://doi.org/10.1063/1.1577829

Mirrorless lasing from nematic liquid crystals in the plane waveguide geometry without refractive index or gain modulation

Applied Physics Letters 89, 031114 (2006); https://doi.org/10.1063/1.2234316

VOLTAGE-DEPENDENT OPTICAL ACTIVITY OF A TWISTED NEMATIC LIQUID CRYSTAL Applied Physics Letters 18, 127 (1971); https://doi.org/10.1063/1.1653593

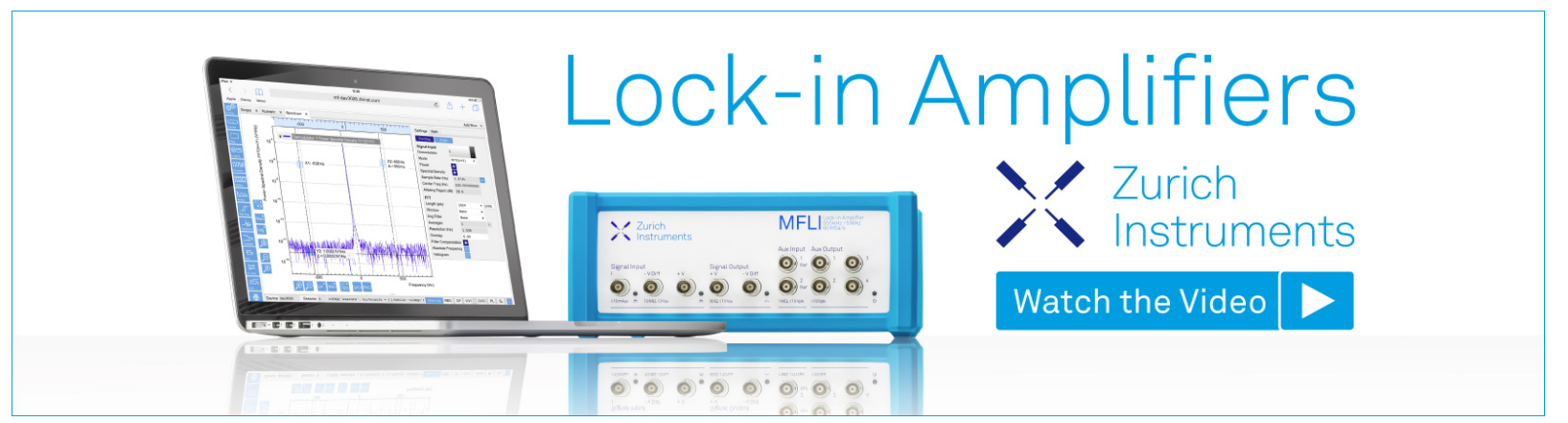




\title{
Electro-tunable laser action in a dye-doped nematic liquid crystal waveguide under holographic excitation
}

\author{
Tatsunosuke Matsui, Masanori Ozaki, ${ }^{a)}$ and Katsumi Yoshino \\ Department of Electronic Engineering, Graduate School of Engineering, Osaka University, \\ 2-1 Yamada-Oka, Suita, Osaka 565-0871, Japan
}

(Received 10 March 2003; accepted 30 May 2003)

\begin{abstract}
Optically pumped distributed feedback lasing has been demonstrated in a dye-doped nematic liquid crystal (NLC) waveguide by holographic excitation. The excitation was performed by two-beam interference using Lloyd mirror configuration. With an applied electric field, continuous tuning of the lasing wavelength was realized due to the change of the effective refractive index of the NLC core layer caused by the reorientation of NLC molecules. (C) 2003 American Institute of Physics. [DOI: $10.1063 / 1.1593827]$
\end{abstract}

In 1971, Kogelnik et al. demonstrated the laser action upon photoexcitation of rhodamine $6 \mathrm{G}$ doped gelatin film with a holographically inscribed phase grating. ${ }^{1}$ In their device, the optical feedback was provided by backward Bragg scattering from phase grating, and mirrorless distributed feedback (DFB) laser action was observed. They also showed the DFB laser action could be achieved by a transient grating using interference fringes induced by two excitation laser beams (holographic excitation). ${ }^{2}$ The wavelength of laser emission $\lambda_{\text {Bragg }}$ upon holographic excitation can be expressed by following equation:

$$
\lambda_{\text {Bragg }}=n_{\text {eff }} \lambda_{\text {ex }} / m \sin \theta,
$$

where $n_{\text {eff }}$ is the effective refractive index of active layer, $\lambda_{\text {ex }}$ is the wavelength of excitation beams, $m$ is the order of diffraction, and $\theta$ is the half-angle between two excitation beams. Based on this equation, the tunability of laser emission wavelength was also demonstrated by changing the angle between two excitation beams $2 \theta$ or refractive index of laser dye solution $n_{\text {eff }}$.

From the equation, it can also be expected that, if the effective refractive index of the active medium $n_{\text {eff }}$ is electrically changed, electrical tuning of laser emission upon holographic excitation can be realized. In this study, we propose an electrical tuning of laser emission using a dye-doped nematic liquid crystal (NLC) as an active medium, as schematically shown in Fig. 1. The coordinate system used in this letter is also indicated in the inset. LC molecules have an elongated rod-like shape, which result in an appearance of an optical anisotropy; that is, LC molecules have an extraordinary refractive index $n_{e}$ along the molecular long axis (director) and an ordinary refractive index $n_{o}$ perpendicular to the director, as shown in the inset of Fig. 1. LC also has anisotropy in dielectric constant, so that the director of LC molecules can be controlled by the applied electric field. ${ }^{3}$ This indicates that, if LC is used as an active material, the effective refractive index of the laser medium $n_{\text {eff }}$ can be electrically controlled due to the field-induced reorientation of LC molecules.

\footnotetext{
${ }^{a)}$ Electronic mail: ozaki@ele.eng.osaka-u.ac.jp
}

The NLC mixture E-44 (Merck) was used as a host material. The dielectric anisotropy of this NLC is positive so that if an electric field is applied to LC layer, LC molecules align their molecular long axis along the applied electric field. As a laser dye dopant in the NLC, [2[2-4-(dimethylamino)phenyl]ethenyl]-6-methyl-4H-pyran-4ylidene] propanedinitrile (Exciton) was used. The concentration of the dye was $0.7 \mathrm{wt} \% . N_{o}$ and $n_{e}$ of this mixture are 1.53 and 1.78 , respectively (at $632.8 \mathrm{~nm}$ ). The sample was inserted in the isotropic phase by capillary action into the sandwiched cell that is composed of two indium-tin-oxidecoated quartz glass substrates. The cell gap was $2.5 \mu \mathrm{m}$. In order to obtain a planarly aligned cell in which the direction of LC initially aligns parallel to the substrates, the surfaces of substrates were coated with poly(vinyl alcohol) (PVA) and rubbed. The PVA layer also acted as a low-index clad layer $\left(n_{\mathrm{PVA}}=1.52\right)$. In such a cell structure, the LC layer acts as a core layer of the slab waveguide and the electromagnetic wave can be confined effectively in the LC layer under the total reflection condition. ${ }^{4,5}$

Figure 2 shows the experimental setup for emission measurement. A third-harmonic light of a Q-switched Nd:YAG laser (Spectra Physics, Quanta-Ray INDI) was used for an excitation, whose wavelength, pulse width, and pulse repetition frequency were $355 \mathrm{~nm}, 8 \mathrm{~ns}$, and $10 \mathrm{~Hz}$, respectively. The polarization of both excitation laser beams was set to be $s$-polarized (along the $y$-axis); that is, perpendicular to the incident plane ( $x z$-plane), in order to make photoinduced transient grating to be pure intensity grating. ${ }^{6,7}$ The excitation laser beams were focused into a stripe using a cylindrical lens. The Al mirror was placed in front of the sample cell

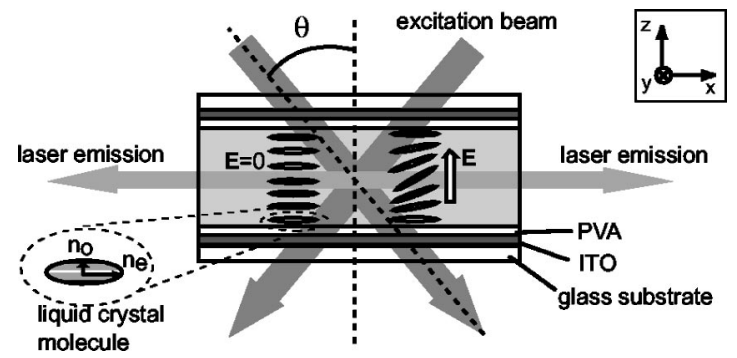

FIG. 1. Schematic representation of the cell structure used in this study for the electrical tuning of the laser emission upon holographic excitation. 


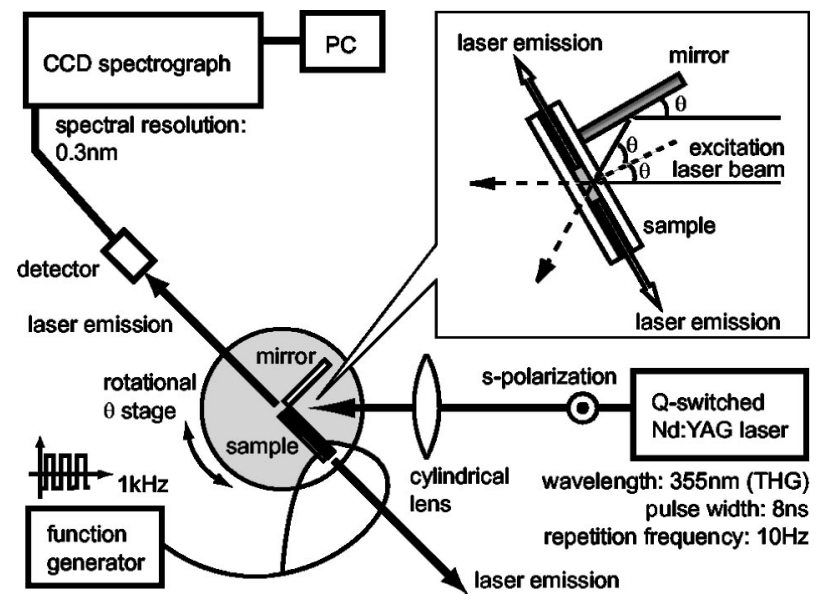

FIG. 2. Schematic representation of the experimental setup for emission measurement. Inset: the Lloyd mirror setup for the interferential illumination.

perpendicular to the cell, so that the so-called Lloyd mirror setup for the holographic illumination was realized, as shown in the inset of Fig. 2. The sample cell and mirror were fixed on a rotational $\theta$ stage, and the angle between two excitation laser beams $2 \theta$ was adjusted by the rotational angle of this stage. In this configuration, the rubbing direction (along the $x$-axis) in the sample cell was perpendicular to the rotational axis (along the $y$-axis) of the stage. That is, the director of NLC molecules in the cell lies in the incident plane of two excitation beams. Under such an illumination condition, both TE and TM guided modes in the NLC core layer feels the ordinary refractive index $n_{o}$ of the NLC in the absence of the electric field. The emission spectra from the sample were measured utilizing a spectrograph with a CCD detector

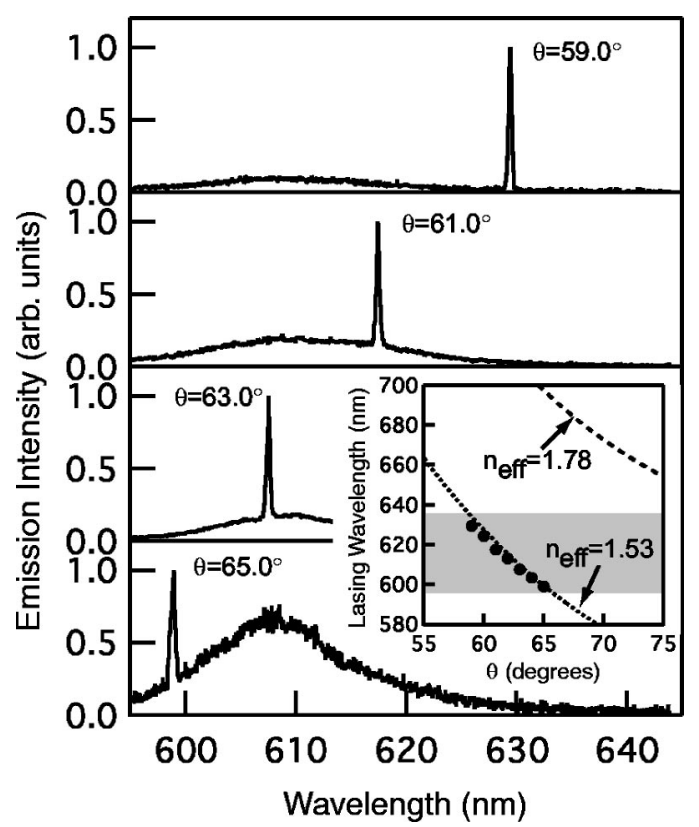

FIG. 3. Emission spectra of the dye-doped NLC waveguide upon holographic excitation at various excitation angles $\left(59.0^{\circ}, 61.0^{\circ}, 63.0^{\circ}\right.$, and $65.0^{\circ}$ ) above the threshold pump pulse energy without applied voltage. Inset: the excitation angle dependence of the lasing wavelength. The shaded region shows the tunable range of this laser. The dashed lines show the theoretical curves of the lasing wavelength under the first-order diffraction condition $(m=1)$ assuming the effective refractive indices $n_{\text {eff }}$ as 1.53 and 1.78 .

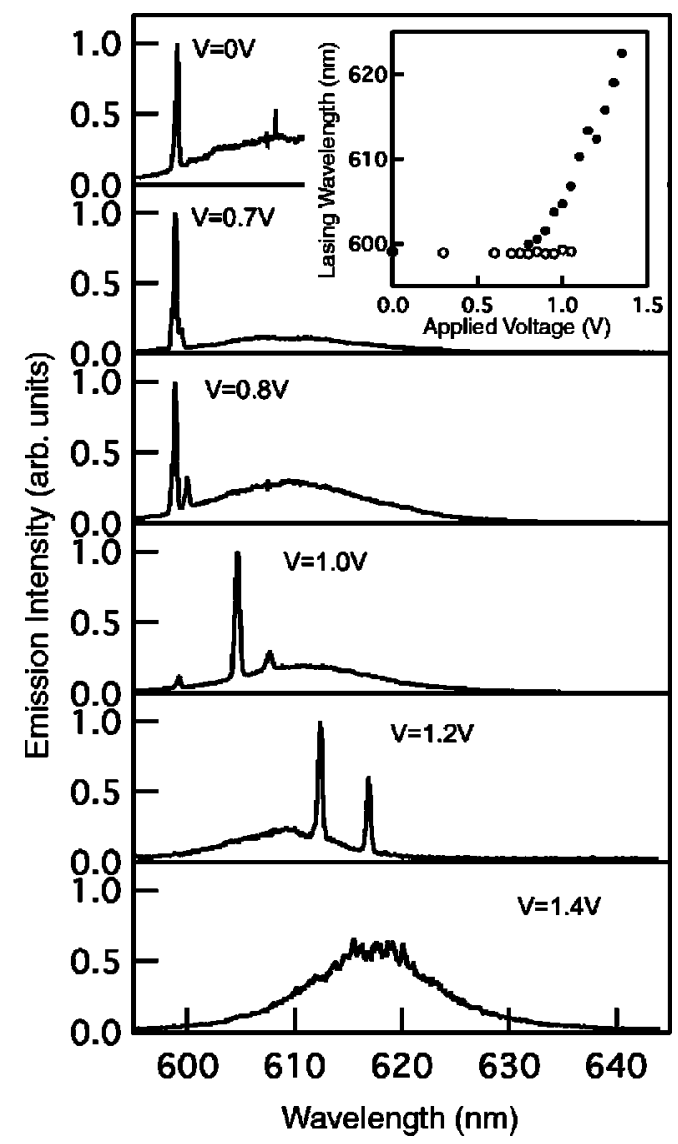

FIG. 4. Emission spectra of the dye-doped NLC waveguide upon holographic excitation at various applied voltages $\left(\theta=65.0^{\circ}\right)$. Inset: the applied voltage dependence of lasing wavelength.

(Oriel, Multi Spec 257) having a spectral resolution of 0.3 $\mathrm{nm}$. For the application of an electric field to the sample cell, a function generator (Hewlett Packard, 3314A) was used. A rectangular shaped ac voltage of $1 \mathrm{kHz}$ was applied.

Figure 3 shows the emission spectra of the dye-doped NLC waveguide for various excitation angles $\left(59.0^{\circ}, 61.0^{\circ}\right.$, $63.0^{\circ}$, and $65.0^{\circ}$ ) above the threshold pump pulse energy without applied voltage. The full width at half-maximum of the emission peak is about $0.5 \mathrm{~nm}$, which is limited by the spectral resolution of our experimental setup. It was shown that the tunability of lasing wavelength upon holographic excitation was about $30 \mathrm{~nm}$. In the inset of Fig. 3, the excitation angle dependence of the lasing wavelength is also shown. The shaded region shows the tunable range of this laser. The dashed lines show the theoretical curves of the lasing wavelength under the first order diffraction condition $(m=1)$ assuming the effective refractive indices $n_{\text {eff }}$ as 1.53 and 1.78. The excitation angle dependence of the lasing wavelength agrees well with the theoretical curve with the effective refractive index $n_{\text {eff }}$ of 1.53 . It was confirmed that the laser emission wavelength could be tuned by changing the excitation angle and that the DFB laser action was realized due to the holographically induced transient grating.

Figure 4 shows the emission spectra of the dye-doped NLC waveguide at various applied voltages. The half-angle between two excitation laser beams $\theta$ was fixed to be $65.0^{\circ}$. When the applied voltage was below $0.7 \mathrm{~V}$, laser emission peak was observed at $599 \mathrm{~nm}$ (peak 1) and no change was observed in the laser emission. When the applied voltage 
exceeded $0.8 \mathrm{~V}$, another new peak (peak 2) appeared in longer wavelength. The peak 2 showed a continuous redshift upon increasing the applied voltage. Above an applied voltage of about $1.0 \mathrm{~V}$, laser emission of peak 2 became multimode. When the applied voltage was more than about $1.4 \mathrm{~V}$, laser emission disappeared. On the other hand, peak 1 did not shift at all. The amplitude of peak 1 decreased gradually, and laser emission peak 1 finally disappeared above $1.1 \mathrm{~V}$. Applied voltage dependences of the lasing wavelength of peak 1 (open circle) and peak 2 (only main peak, closed circle) are summarized in the inset of Fig. 4. The threshold voltage for the appearance of peak 2 is observed at about 0.8 $\mathrm{V}$, which should be attributed to the Frederiks transition of the NLC. The electrical tuning of laser action could be performed reversibly.

In order to explain these results, TE and TM guided modes should be treated separately. The reorientation of NLC molecules upon the application of an electric field takes place in the $x z$-plane, so that the effective refractive index $n_{\text {eff }}$ for TM guided mode increases gradually with the fieldinduced reorientation of the NLC, the lasing wavelength associated with TM guided mode should shift to a longer wavelength. Consequently, the redshift of the lasing peak 2 might be attributed to TM guided mode. With the increase of the effective refractive index $n_{\text {eff }}$, the effective thickness of the core layer also increases and higher-order guided mode should appear. The appearance of multi-mode emission in peak 2 upon application of more than about $1.0 \mathrm{~V}$ can be explained in terms of this higher order guided mode. At more than $1.4 \mathrm{~V}$, the diffraction condition for the DFB laser action could not be satisfied in the tunable range of this laser anymore, so that the laser action was not observed.

On the other hand, the TE guided mode feels the ordinary refractive index $n_{o}$ of the NLC regardless of the applied voltage, so that no change in lasing wavelength of peak 1 should be attributed to TE guided mode. Although no change of lasing wavelength was observed, the lasing intensity of peak 1 was reduced and peak 1 disappeared at more than about 1.1 V. This is attributed to the redshift of the photoluminescence (PL) spectrum of the doped laser dye caused by the increase in the effective refractive index of the NLC core, which can also be seen in Fig 4. Due to the shift of the PL spectrum, attenuation of optical gain was caused.
As mentioned earlier, the tuning of the lasing wavelength was realized slightly above the threshold voltage. From the shift of lasing wavelength of $30 \mathrm{~nm}$, the increase in the effective refractive index of the core layer can be estimated as 0.08 from the diffraction condition indicated earlier. This value corresponds to the $37.5^{\circ}$ tilt of the NLC molecules assuming a homogeneous deformation of NLC upon the application of the electric field. From this estimation, it can be considered that the tuning of this laser was performed using small deformation, and lasing properties is quite sensitive to the deformation of NLC alignment. Using this holographic excitation technique, fabrication of submicrometer sized periodic structure for the DFB laser action is not necessary, and the bulky fluidic characteristic of LC can be effectively utilized. By varying cell configurations such as cell gap, initial alignment of LC, switching procedure of LC, and so on, various lasing modes based on the waveguiding mode theory can be realized and controlled.

In conclusion, optically pumped DFB laser action was observed in a dye-doped NLC waveguide. The DFB laser action was realized by holographic excitation using Lloyd mirror setup. Using an applied electric field, the wavelength of the laser emission was continuously tuned on the basis of the reorientation of NLC molecules. For the fabrication of this device, the industrially established procedures for making LC displays can be adopted, and monolithic integration into the optical integrated circuits can also be expected as an electrically tunable light source.

This work is supported in part by a Grant-in-Aid for Scientific Research (14350165) from the Japan Ministry of Education, Culture, Sports, Science and Technology.

${ }^{1}$ H. Kogelnik and C. V. Shank, Appl. Phys. Lett. 18, 152 (1971).

${ }^{2}$ C. V. Shank, J. E. Bjorkholm, and H. Kogelnik, Appl. Phys. Lett. 18, 395 (1971).

${ }^{3}$ P. G. deGennes and J. Prost, The Physics of Liquid Crystals (Oxford University Press, New York, 1993).

${ }^{4}$ D. J. Channin, Appl. Phys. Lett. 22, 365 (1973).

${ }^{5}$ J. P. Sheridan and T. G. Giallorenzi, J. Appl. Phys. 45, 5160 (1974).

${ }^{6}$ H. J. Eichler, P. Gunter, and D. W. Pohl, Laser Induced Dynamic Gratings (Springer, Berlin, 1985).

${ }^{7}$ N. K. Viswanathan, S. Balasubramanian, L. Li, S. K. Tripathy, and J. Kumar, Jpn. J. Appl. Phys. 38, 5928 (1999). 